\title{
Analysis of the levels of hope and influencing factors in infertile women with first-time and repeated IVF-ET cycles
}

Ying $\mathrm{Ni}^{1,3+}$, Limin Huang ${ }^{2+}$, Chenye Tong ${ }^{2}$, Wen Qian ${ }^{2}$ and Qiong Fang ${ }^{1,3 *}$

\begin{abstract}
Purpose: To explore the hope levels and influencing factors in infertile women undergoing first-time and repeated in vitro fertilization-embryo transfer (IVF-ET) cycles.

Methods: This study was a cross-sectional and convenient sampling study conducted among patients undergoing IVF-ET from January to June 2019. Patients were divided into first-time and repeated groups by the number of IVF-ET cycles, and then a questionnaire survey was administered. The questionnaire included demographic information, Herth hope index $(\mathrm{HHI})$ scale, Locke-Wallace short marital-adjustment test scale and social support rating scale. Multiple linear regression was used to analyse the influencing factors associated with hope levels.
\end{abstract}

Results: A total of 298 IVF-ET patients were recruited for the study, including 150 (50.3\%) in the first-time cycle group and $148(49.7 \%)$ in the repeated cycle group. The HHI score of the repeated cycle group was significantly lower than that of the first-time cycle group ( $34.4 \pm 3.5$ vs. $37.5 \pm 3.7, P<0.001)$. Multiple linear regression analysis indicated that repeated IVF-ET and age were independently negatively correlated with $\mathrm{HHI}$, with standardized coefficient $\beta$ values of -0.895 and -0.223 , respectively (both $P<0.001)$. High education level $(P=0.002)$, high monthly income $(P=0.020)$, high degree of short marital-adjustment test $(P<0.001)$ and social support rating $(P<0.001)$ were independently positively correlated with $\mathrm{HHI}$.

Conclusion: Infertile women undergoing repeated IVF-ET have low hope levels. Maintaining a good marriage adjustment and establishing a good social support and relationship network could effectively improve their hope levels.

\section{Plain language summary}

Hope level is closely related to patients' mental health and emotional state. Therefore, improving the hope level of infertile women with IVF-ET could contribute to relieving the psychological pressure of patients and improving the success rate of pregnancy. This study aimed to evaluate the hope level of infertile women with different IVF-ET cycles and analyse its influencing factors to provide guidance for clinical nurses to clarify the intervention objects, select reasonable intervention measures and improve the hope levels of patients. A total of 298 IVF-ET patients were recruited in the study, including 150 in the first-time cycle group and 148 in the repeated cycle group. The results showed that the hope level of patients with repeated IVF-ET cycles was significantly lower than that of patients undergoing

\footnotetext{
*Correspondence: ny21085@outlook.com

${ }^{\dagger}$ Ying Ni and Limin Huang have contributed equally to this work

${ }^{1}$ Department of Nursing, Ruijin Hospital, Shanghai Jiaotong University

School of Medicine, Shanghai, China

Full list of author information is available at the end of the article
}

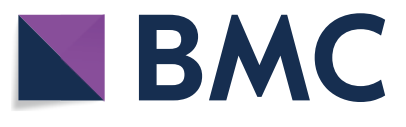

(c) The Author(s) 2021. Open Access This article is licensed under a Creative Commons Attribution 4.0 International License, which permits use, sharing, adaptation, distribution and reproduction in any medium or format, as long as you give appropriate credit to the original author(s) and the source, provide a link to the Creative Commons licence, and indicate if changes were made. The images or other third party material in this article are included in the article's Creative Commons licence, unless indicated otherwise in a credit line to the material. If material is not included in the article's Creative Commons licence and your intended use is not permitted by statutory regulation or exceeds the permitted use, you will need to obtain permission directly from the copyright holder. To view a copy of this licence, visit http://creativecommons.org/licenses/by/4.0/. The Creative Commons Public Domain Dedication waiver (http://creativeco mmons.org/publicdomain/zero/1.0/) applies to the data made available in this article, unless otherwise stated in a credit line to the data. 
first-time treatment. We also analysed its influencing factors and found that repeated IVF-ET cycles and age were independent negative correlation factors in hope levels. High monthly income, living in the city, high marital adjustment and social support score were independent positive correlation factors in hope levels. Therefore, maintaining a good marriage adjustment and establishing a good social support and relationship network could effectively improve their hope levels.

Keywords: In vitro fertilization-embryo transfer, Repeated cycles, Hope level, Influencing factors

\section{Introduction}

With changes in the living environment, the accelerating pace of life and delays in marriage and childbearing, the incidence of infertility is rising year by year. According to statistics from the World Health Organization (WHO), approximately $8-12 \%$ [1] of couples worldwide have experienced infertility. As consistently demonstrated, infertility has a strong and negative impact in several areas of the individual's life. For instance, impairments in marital relationships $[2,3]$, sexual satisfaction $[4,5]$, psychosocial well-being [6-8] and psychological correlates $[9,10]$ are significantly associated with infertility.

Since the approaches of controlled ovarian stimulation (COS) and the conditions of the embryonic laboratory have improved, in vitro fertilization-embryo transfer (IVF-ET), as the main assisted reproductive therapy for infertile patients, has brought hope to many infertile patients. However, due to the long treatment cycle, frequent visits, high costs, various traumatic operations and uncertain results, IVF-ET is a process with hope and disappointment, exerting brings both physical and psychological pressure on infertile women [11].

Some patients experience repeated implantation failure, and approximately $25 \%$ had already undergone more than five IVF-ET cycles [12]. Repeated IVF-ET significantly increases the risk of ovarian hyperstimulation syndrome, premature delivery and oestrogen-dependent tumours [41-43]. The study also found that anxiety and stress are present in women throughout the treatment [13]. At the same time, the negative emotions caused by repeated IVF-ET failure can further reduce the success rate of pregnancy [14].

Hope is a valuable human response that has received increasing attention in recent years $[15,16]$. Hope is a type of multidimensional positive life power and provides an optimistic expectation of a wonderful outcome and an effective adjustment mechanism that enables individuals to overcome current difficulties [17]. Hope can change the experiences of patients with chronic diseases and their lifestyles [18]. Hope has a beneficial effect on individuals' health because it enables them to cope with conflicts, achieve healthy objectives, maintain quality of life, and promote health [19, 20]. Rahimipour et al. (2015) conducted a clinical trial on the effect of hope therapy on anxiety, depression and stress among 50 patients undergoing hemodialysis [21]. They found a significant reduction in mean scores of anxiety, depression and stress in the hope therapy group before and after the intervention, and changes in mean scores of depression, anxiety and stress were lower in the hope therapy group than in the placebo group. Hope level is closely related to patients' mental health and emotional state. Therefore, improving the hope level of infertile women with IVF-ET could contribute to relieving the psychological pressure of patients and improving the success rate of pregnancy. In the current literature, exploration of hope and its role among infertile women with IVF-ET has not been widely undertaken in this respect. This study aimed to use the Hertz hope index (HHI) [23] to evaluate the hope levels of infertile women with different IVF-ET cycles and analyse its influencing factors to provide guidance for clinical nurses to clarify the intervention objects, select reasonable intervention measures and improve the hope levels of patients.

\section{Methods}

\section{Ethics statement}

The study protocol was in accordance with ethical standards and was approved by the Ethics Committee of Shanghai Ruijin Hospital. Written informed consent was obtained from each participant. Information collected from all participants was kept confidential and anonymous.

\section{Data and study design}

This study was a cross-sectional and convenient sampling study conducted among outpatient women diagnosed with infertility and treated with IVF-ET cycles from January to June 2019. All of the participants were recruited at the Reproductive Medical Center, Ruijin Hospital, Shanghai Jiaotong University School of Medicine in China. The inclusion criteria were as follows: (1) female outpatients diagnosed with infertility and $\geq 1$ IVF-ET cycle; (2) women who signed informed consent forms after completely comprehending the content of the study; and (3) women who had the basic ability to read, communicate, and complete the questionnaire independently. The exclusion criteria were as follows: (1) 
women diagnosed with previous or current mental disorders, cognitive impairment, or inability to understand the content of the questionnaire; (2) women diagnosed with severe chronic diseases; and (3) women who had the embryos from previous cycles successfully implanted. The participants were divided into two groups according to the number of IVF-ET cycles. In the first-time group, participants underwent the first IVF-ET cycle. In the repeated group, each participant underwent 2 or more IVF-ET cycles. The investigation was conducted during the last follow-up period before the next cycle. After written informed consent was obtained for this study, a self-report questionnaire was distributed to each eligible participant, and clinical data were collected from their medical records. Questionnaires with any missing data were excluded from statistical analyses. All questionnaires were required to be completed independently by the patients. The questionnaires were collected, and their completeness was checked at that time. The investigation process was conducted anonymously. The questionnaire elimination criteria were as follows: (1) the same answer for each question; or (2) withdrawal from this research for various reasons.

The sample size estimations were performed using the formula $\mathrm{n}=\mathrm{Z}_{1-\alpha / 2}{ }^{2} * \mathrm{SD}^{2} / \mathrm{d}^{2}$ [22] with a $95 \%$ confidence interval. Based on a published study [26], we chose 5.57 as the standard deviation of the hope level within patients undergoing IVF treatment and 1.0 for the accepted margin of error. It was estimated from the sample size calculation that 240 participants (120 in each group) would meet the requirement. Considering the chances of a $20 \%$ dropout rate, we decided to recruit a minimum of 290 participants ( $\mathrm{n}=145$ in each group).

A total of 315 questionnaires were distributed, and all of them were received, for a response rate of $100 \%$. Seventeen questionnaires were excluded: 2 for missing answers, 10 for the same answers for each question, and 5 for deviation from the inclusion criteria. In total, 298 complete responses were received in the present study. The effective rate of the questionnaires was $94.6 \%$.

\section{Measures}

\section{Demographic characteristics}

The demographic characteristics were collected with a general information questionnaire designed by our panel, which included age, marriage age, residence, occupation, education level, monthly household income, and number of treatment cycles.

\section{Measurement of hope level}

This study used the Herth hope index (HHI) scale [23] to measure the hope level. The HHI scale consists of three dimensions: a positive attitude towards reality and the future (including Items 1, 2, 6 and 11); taking positive action (including Items 4, 7, 10 and 12); and maintaining a close relationship with others (including Items 3, 5, 8 and 9). All items were scored using a 4-level Likert scoring method with 1-4 points representing very opposed, opposed, agree and very much agree, respectively. The total score of the scale is $12-48$ points, among which the low, medium and high hope levels are 12-23 points, 24-35 points and 36-48 points, respectively. The higher that the score is, the higher that the level of hope is of the respondent. The Cronbach's $\alpha$ value of the reliability of the scale was 0.85 [23].

\section{Measurement of marriage moderation}

The Locke Wallace short marital adjustment test (MAT), jointly developed by Locke and Wallace, was used to assess marriage moderation. The MAT scale is designed to objectively and quantitatively assess the individual's adaptability and satisfaction with the relationship between husband and wife [24]. The scale consists of 15 items, reflecting the adjustment of marriage from 15 aspects. The total score ranges from 2 to 158 . The higher that the score is, the better that the marriage adjustment is, and the higher that the quality of the marriage is. If the score is less than 100 , it means that the marriage is maladjusted. If the score is more than 100 , it means that the marriage has adapted well. The reliability coefficient of the questionnaire was 0.90 , and the discriminant ratio was 17.5 , indicating good reliability and validity.

\section{Measurement of social support}

The Chinese version of the Social Support Rating Scale (SSRS), designed by Xiao Shuiyuan, was used to assess social support [25]. The scale has 10 items, including objective support (3 items), subjective support (4 items) and support utilization ( 3 items). The total score range is 12-66. The higher that the score is, the more support that there is. The consistency of the total score was 0.92, and the consistency of each item was $0.89-0.94$.

\section{Statistical analysis}

In this study, statistical analysis was performed with SPSS software, version 23.0. The measurement data are presented as the mean \pm standard deviation, and the enumeration data are expressed as the frequency and constituent ratio (\%). Student's t test was used to compare the two groups. The $\mathrm{x}^{2}$ test was used to test the rate inspection. Pearson's correlation analysis (r) was used to analyse the correlations of continuous variables or rank data. Stepwise analysis was used for multivariate analysis. Retest reliability was tested by consistency $\mathrm{\kappa}$ value representation. The validity was analysed by principal 
component analysis, factor analysis and principal component analysis. $\mathrm{P}<0.05$ was considered statistically significant.

\section{Results}

\section{Description of the participants}

There were 150 patients in the first-time group out of 298 participants, while the 148 in the repeated group had 53 with two IVF-ET cycles, 51 with three cycles, and 44 with four or more cycles. The age and marriage age of the patients in the repeated group were $33.0 \pm 3.9$ years old and $6.7 \pm 3.9$ years, respectively, which were significantly higher than those in the first-time group $(31.9 \pm 4.3$ years old and $5.8 \pm 3.9$ years, respectively) $(\mathrm{P}<0.001)$. The results showed that the age of patients with repeated IVF-ET was higher and the marriage age was old. Conversely, comparing the two groups, there were no significant differences in education level, permanent residence, occupation or family monthly income between the two groups $(\mathrm{P}>0.05)$; see Table 1 .

\section{Reliability and validity of $\mathrm{HHI}$ scale}

The reliability and validity of the HHI scale were analysed before it was used in the participants of this study. Fifty patients were retested after 2 weeks, and the consistency test results indicated good test-retest reliability with $k=0.805(P<0.05)$. The patient data were analysed by principal component analysis. The results of the suitability test showed that the KMO statistical value was 0.765 $(P=0.01)$, indicating that factor analysis was suitable. The correlation coefficient matrix was rotated with maximum variance to extract the factors with eigenvalues $>1$.
The eigenvalues of the three principal components were $2.365,1.427$ and 1.119 . The cumulative variance contribution rate was $69.4 \%$. The factor components produced by statistical analysis were consistent with the theoretical structure.

\section{Hope level, marriage adjustment and social support}

The HHI, MAT and SSRS scores in the repeated group were $34.4 \pm 3.5,99.0 \pm 24.9$ and $35.4 \pm 7.2$, respectively, which were significantly lower than $37.5 \pm 3.7$, $116.3 \pm 22.4$ and $39.5 \pm 5.2$ in the first-time group $(\mathrm{P}<0.001)$; see Fig. 1. The hope level, marital adjustment and social support of patients with repeated IVF-ET were significantly decreased.

Comparing the scores of the three dimensions of the $\mathrm{HHI}$ also showed that the scores in the repeated group were significantly lower than those of the first-time group (see Table 2). According to the scores, they were divided into low-level, medium-level and high-level subgroups. Further analysis showed that $67.3 \%(n=101)$ of the patients in the first-time group had high levels. However, the high level was only $29.7 \%(\mathrm{n}=44)$ in the repeated group $(P<0.001)$. More than half of the patients undergoing first-time IVF-ET were at a high hope level, while the proportion of patients with a high hope level in the repeated group was significantly decreased, as shown in Table 2.

\section{Correlation analysis of influencing factors of hope level}

The correlations between of the HHI with demographic characteristics, MAT and SSRS were analysed. The results showed that the HHI was positively correlated with MAT

Table 1 Demographic characteristics of infertile women undergoing IVF-ET with different cycles

\begin{tabular}{|c|c|c|c|c|c|}
\hline Variables & Total $(n=298)$ & $\begin{array}{l}\text { First-time group } \\
(n=150)\end{array}$ & $\begin{array}{l}\text { Repeated group } \\
(n=148)\end{array}$ & $t / x^{2}$ & $P$ \\
\hline Age (years) & $31.9 \pm 4.3$ & $31.0 \pm 4.4$ & $33.0 \pm 3.9$ & -4.175 & $<0.001$ \\
\hline Marriage age (years) & $5.8 \pm 3.9$ & $4.8 \pm 3.7$ & $6.7 \pm 3.9$ & -4.165 & $<0.001$ \\
\hline \multicolumn{6}{|l|}{ Educational level, n (\%) } \\
\hline Junior high school or below & $56(18.8)$ & $28(18.7)$ & $28(18.9)$ & 0.003 & 0.956 \\
\hline Senior high school or above & $242(81.2)$ & $122(81.3)$ & $120(81.1)$ & & \\
\hline \multicolumn{6}{|l|}{ Residence, n (\%) } \\
\hline Rural & $67(24.2)$ & $36(24.2)$ & $31(24.2)$ & 0.118 & 0.731 \\
\hline City/town & $210(75.8)$ & $113(75.8)$ & $97(75.8)$ & & \\
\hline \multicolumn{6}{|l|}{ Occupation, n (\%) } \\
\hline Physical & $60(20.1)$ & 28(18.7) & $32(21.6)$ & 0.405 & 0.525 \\
\hline Mental & 238(79.9) & $122(81.3)$ & $116(78.4)$ & & \\
\hline \multicolumn{6}{|c|}{ Family monthly income (yuan), n (\%) } \\
\hline$<5000$ & $112(37.6)$ & $55(36.7)$ & $57(38.5)$ & 4.879 & 0.087 \\
\hline $5000-10,000$ & $75(25.2)$ & $31(20.7)$ & $44(29.7)$ & & \\
\hline$\geq 10,000$ & $111(37.2)$ & $64(42.6)$ & $47(31.8)$ & & \\
\hline
\end{tabular}




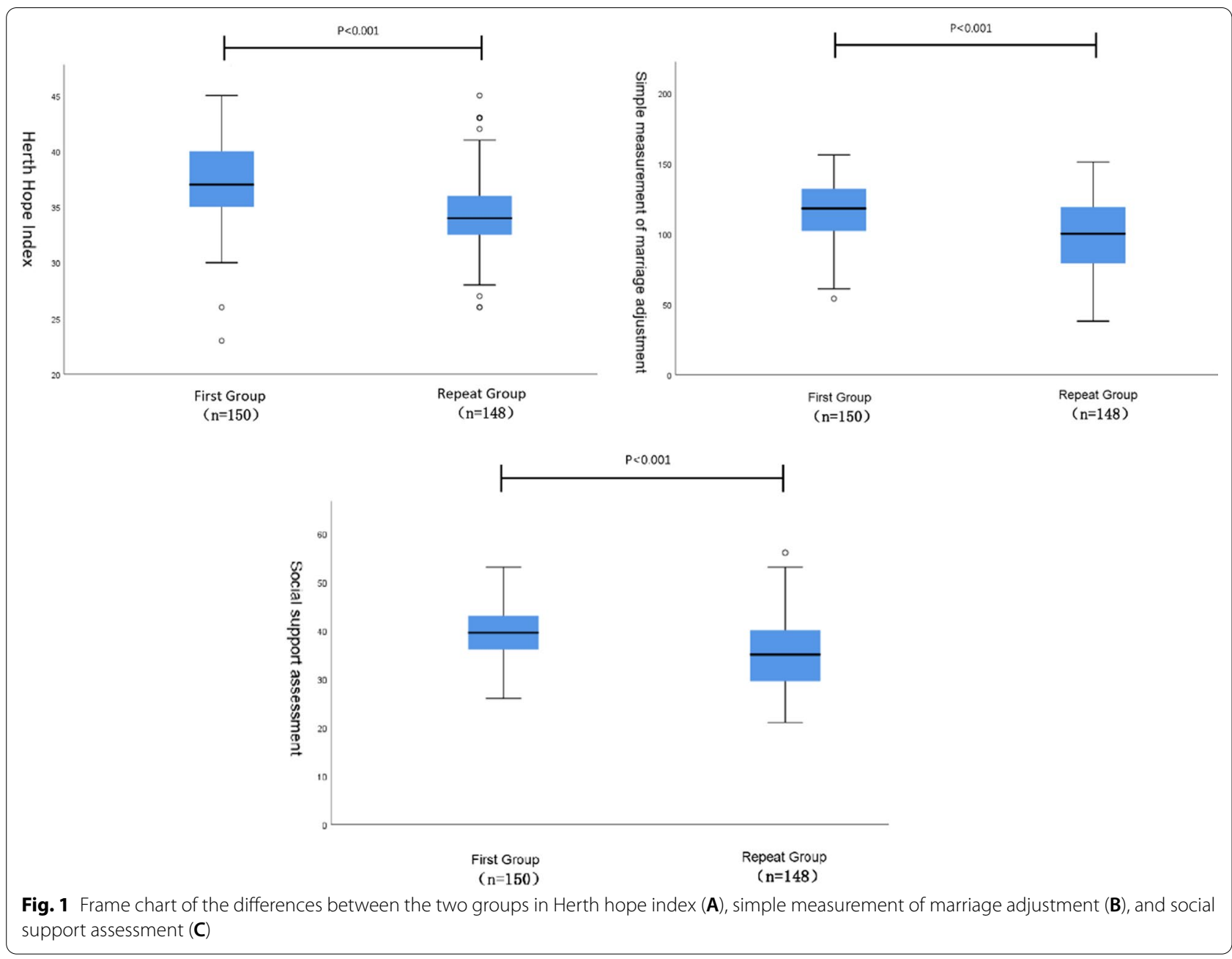

Table $2 \mathrm{HHI}$ score in dimensions and subgroups of infertile women undergoing IVF-ET

\begin{tabular}{|c|c|c|c|c|c|}
\hline & Total $(n=298)$ & $\begin{array}{l}\text { First-time group } \\
(n=150)\end{array}$ & $\begin{array}{l}\text { Repeated group } \\
(n=148)\end{array}$ & $t / x^{2}$ & $\mathbf{P}$ \\
\hline Herth hope index total score & $35.9 \pm 3.9$ & $37.5 \pm 3.7$ & $34.4 \pm 3.5$ & 7.391 & $<0.001$ \\
\hline Positive attitude towards reality and future & $11.9 \pm 1.4$ & $12.3 \pm 1.4$ & $11.5 \pm 1.4$ & 4.930 & $<0.001$ \\
\hline Positive actions taken & $11.7 \pm 1.6$ & $12.1 \pm 1.6$ & $11.3 \pm 1.4$ & 4.373 & $<0.001$ \\
\hline Keep a close relationship with others & $12.3 \pm 1.9$ & $13.1 \pm 1.7$ & $11.5 \pm 1.7$ & 7.761 & $<0.001$ \\
\hline $\begin{array}{l}\text { Low level } \\
\mathrm{n}(\%)\end{array}$ & $\begin{array}{l}1 \\
(0.3 \%)\end{array}$ & $\begin{array}{l}1 \\
(0.7 \%)\end{array}$ & $\begin{array}{l}0 \\
(0.0 \%)\end{array}$ & 45.521 & $<0.001^{*}$ \\
\hline $\begin{array}{l}\text { Medium level } \\
\mathrm{n}(\%)\end{array}$ & $\begin{array}{l}152 \\
(51.0 \%)\end{array}$ & $\begin{array}{l}48 \\
(32.0 \%)\end{array}$ & $\begin{array}{l}104 \\
(70.3 \%)\end{array}$ & & \\
\hline $\begin{array}{l}\text { High level } \\
\mathrm{n}(\%)\end{array}$ & $\begin{array}{l}145 \\
(48.7 \%)\end{array}$ & $\begin{array}{l}101 \\
(67.3 \%)\end{array}$ & $\begin{array}{l}44 \\
(29.7 \%)\end{array}$ & & \\
\hline
\end{tabular}

*P value is the result of likelihood ratio

$(\mathrm{r}=0.565, \mathrm{P}<0.01)$ (see Fig. 2a), SSRs $(\mathrm{r}=0.498, \mathrm{P}<0.01)$ (see Fig. $2 b)$, monthly income $(r=0.269, P<0.01)$, education level $(\mathrm{r}=0.292, \mathrm{P}<0.01)$, permanent residence $(\mathrm{r}=0.189, \mathrm{P}<0.01)$ and mental work $(\mathrm{r}=0.179, \mathrm{P}<0.01)$.
Conversely, the HHI was negatively correlated with age $(r=-0.204, P<0.01)$, marital age $(r=-0.126$, $\mathrm{P}=0.029)$ and repeated IVF-ET $(\mathrm{r}=-0.480, \mathrm{P}<0.01)$. It was indicated that the hope level of older, long-term 

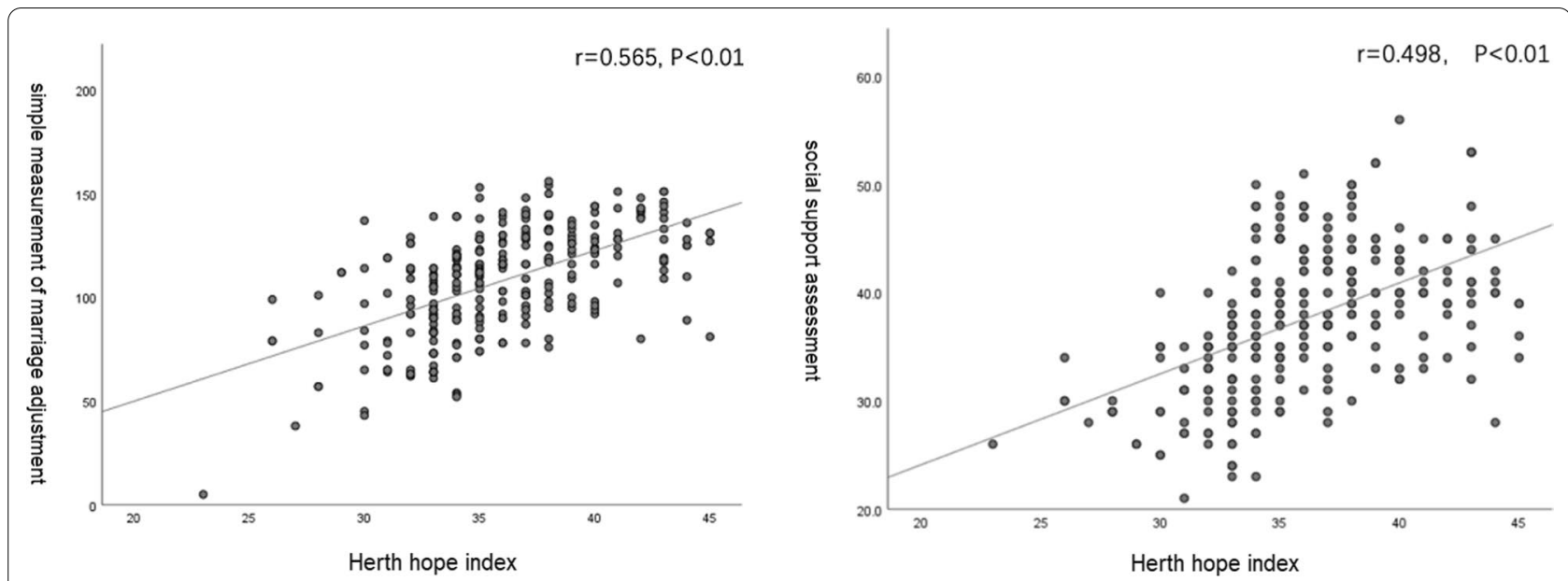

Fig. 2 Correlation analysis of HHI with MAT and SSRS

married patients and cases with repeated IVF-ET treatment was low.

\section{Multiple linear regression analysis on the influencing factors of hope level}

HHI was used as the dependent variable, and the variables with statistical significance in univariate analysis were included in the multiple linear regression model (stepwise regression). As shown in Table 3, the results showed that repeated IVF-ET $(\beta=-0.859, \mathrm{P}<0.001)$ and age $(\beta=-0.223, \mathrm{P}<0.001)$ was negatively correlated with hope levels. However, education level, monthly income, marital age, and MAT and SSRS scores were positively correlated with the HHI. Repeated IVF-ET and age were independent factors of HHI downregulation.

\section{Discussion}

Hope is an individual's confidence and expectations to solve problems when facing difficulties. Patients with a high hope level can actively seek help and solve problems. Hope is believed to be a psychological force guiding patients to manage diseases more actively [26]. Currently, psychological research on infertile women with IVF-ET mainly focuses on anxiety, depression and stress. However, to the best of our knowledge, there has been little research focused on their hope levels. Therefore, this study conducted a cross-sectional study on the hope level and its influencing factors in infertile women undergoing IVF-ET. This study found that the HHI scores of patients with repeated IVF-ET cycles were significantly lower than that of those with first-time treatment $(32.0 \pm 3.3$ vs. $36.0 \pm 3.1, \mathrm{P}<0.001)$. The scores of positive attitudes towards reality and the future, positive action taken and close relationships with others all decreased remarkably. In the repeated group, the proportion of the high-level subgroup was only $11 \%$, which was significantly lower than the $58.5 \%$ in the first-time group. Multivariate analysis showed that age and repeated IVF-ET cycles were independent negative correlation factors of hope level. High monthly income, living in the city, high marital adjustment and high social support score were independent positive

Table 3 Multivariate linear regression analysis of influencing factors of $\mathrm{HHI}$ in infertile women undergoing IVF-ET

\begin{tabular}{|c|c|c|c|c|c|c|}
\hline Variables & $\begin{array}{l}\text { Non standardized } \\
\text { coefficient B }\end{array}$ & Standard error & $\begin{array}{l}\text { Standardized } \\
\text { coefficient } \beta\end{array}$ & $\mathbf{t}$ & $\mathbf{P}$ & $95 \%$ Confidence interval \\
\hline Constant & 30.288 & 2.066 & - & 14.664 & $<0.001$ & $26.223 \sim 34.353$ \\
\hline Repeated IVF-ET & -0.859 & 0.141 & -00.291 & -6.095 & $<0.001$ & $-1.136 \sim-0.581$ \\
\hline MAT & 0.045 & 0.009 & 0.288 & 5.266 & $<0.001$ & $0.028 \sim 0.062$ \\
\hline SSRS & 0.117 & 0.031 & 0.198 & 3.802 & $<0.001$ & $0.057 \sim 0.178$ \\
\hline Age & -0.223 & 0.059 & -0.242 & -3.779 & $<0.001$ & $-0.339 \sim-0.107$ \\
\hline Marriage age & 0.275 & 0.064 & 0.274 & 4.306 & $<0.001$ & $0.150 \sim 0.401$ \\
\hline Monthly income & 0.516 & 0.220 & 0.114 & 2.349 & 0.020 & $0.084 \sim 0.949$ \\
\hline Education level & 1.474 & 0.478 & 0.147 & 3.081 & 0.002 & $0.532 \sim 2.416$ \\
\hline
\end{tabular}

$\mathrm{R}^{2}=0.487$, adjusted $\mathrm{R} 2=0.475, \mathrm{P}<0.05$ 
correlation factors with hope level. The hope level score obtained by this research was lower than that obtained by TANG Nan et al. [26]. The reason could be that more infertile women with repeated IVF-ET cycles were included in this study. Among them, patients with more than 4 cycles accounted for $12.7 \%$, and the score of these patients was significantly lower than that in the first-time group. We found that infertile women with repeated IVF-ET cycles were the main objects of nursing interventions. Maintaining good marital adjustment and establishing good social support and relationship networks might be effective ways to improve the hope levels of infertile women with IVF-ET.

In our previous study [27], we found that the psychological pressure of infertile women undergoing IVF-ET mainly comes from worries about age and pregnancy success rates, family, society and the surrounding environment. Therefore, we not only evaluated the level of hope but also conducted a targeted questionnaire survey on marriage adjustment and social support. Some studies have found that hope is positively correlated with happiness, persistence, health, social support, improvement of mental pressure and other factors [28]. Hope is an important factor in helping patients to recover when they feel stressed. This study also found that patients undergoing first-time IVF-ET had a better level of hope. These patients had clear treatment plans and great expectations for success. However, once the first cycle fails, the psychological pressure of patients increases significantly, leading to a significant decline in the level of hope.

A study from Israel found that, although the overall success rate of IVF-ET reached $54 \%$, approximately $25 \%$ of the patients underwent more than five treatment cycles, and $12 \%$ of the patients underwent more than seven cycles [12]. Some studies have indicated that ovarian reserve function decreased and the risk of women reaching menopausal transition or menopause ahead of time increased significantly during repeated IVF-ET cycles, especially more than three cycles [44, 45]. Repeated IVFET failure easily causes anxiety, depression and other adverse psychological problems [29]. This study also found that repeated IVF-ET cycles were an independent negative correlation factor for the decline in hope level. Therefore, in nursing care, patients must have an objective understanding of the outcome of repeated IVF-ET cycles. Nursing staff must provide appropriate guidance and suggestions, offer more disease-related knowledge, and prevent patients from having unrealistic expectations about treatment. Based on the three dimensions of hope levels, there is one factor of "keeping close with others", in addition to other personal factors. It is easy to find the status of social and marital support of patients with the help of the HHI scale. It is also recommended that reproductive clinics guide patients to obtain more support from spouses, friends, family and so on.

This study also found that the age of patients in the repeated group was significantly higher than that in the first-time group. This finding showed that elderly patients might experience more IVF-ET cycles to obtain clinical pregnancy. The success rate of older pregnant women with first-time IVF-EF is low due to decreases in poor endometrial receptivity, immune dysfunction, embryo defects and other factors [30]. Clinical studies have found that the more that the age increases -40 and over - the more that the FSH levels increase, and the more that the quality of embryos decreases (Aneuploidy) [31]. Long marital age is also an obvious characteristic of the repeated group. Women with shorter marriages have closer relationships with their husbands and will receive more support from their husbands. Long-term married couples will face more psychological pressure with repeated failures in long-term treatment. Li et al. [32] found that, in 260 pairs of infertile couples treated with IVF-ET, the incidence of psychological problems, such as anxiety and depression, was significantly higher than that in normal fertile couples. Infertile women undergoing repeated IVF-ET cycles often bear the dual pressures of family and society and have varying degrees of psychological problems. Additionally, in our previous study, we found that the anxiety and depression of patients with repeated implantation failure has significantly affected fertility quality of life [33]. In the process of treatment, negative emotions often affect the outcomes of pregnancy. Appropriate psychological care can help to relieve anxiety, tension and other emotions and improve the success rate of IVF-ET treatment [34]. Therefore, with older and longer married couples, we must inform them of the adverse side effects of repeated IVF-ET treatments and perform more psychological nursing. Nursing staff can act as the mediator of patients' marriages, encourage husbands to listen more and care more about patients' state and relieve patients' anxiety and tension to improve patients' hope levels. Some studies have also found that shared decision-making interventions can improve the psychological state of patients undergoing IVF-ET, improve their hope levels and treatment compliance, and significantly improve the pregnancy rate [35]. Helping patients to establish a sense of gratitude is helpful to alleviating the anxiety of patients in the process of IVF-ET [36]. This study was only a cross-sectional analysis, and we should try more interventions in the future.

\section{Limitations}

This study had a few limitations. First, the convenience sampling method was adopted in this study, which might have affected the representation of the results 
to a certain extent. Second, the HHI has been more commonly used to evaluate the hope levels of cancer patients [37]. A systematic analysis of hope scores found that, in 68 studies, the Snyder Hope scale (46\%) was the most commonly used, followed by the $\mathrm{HHI}$ [38]. Further studies using more scales, such as the Beck Scale [39], could be helpful to evaluate the level of hope more richly. Further studies must be conducted to examine whether the results of the present study are suitable for different cultural contexts. Moreover, there could still be residual variables that were not considered. Zhou [40] measured salivary amylase levels to reflect the stress states of patients. Through objective comparison, it is speculated that a high pressure level is also related to IVF-ET failure. Therefore, it deserves to be further explored.

\section{Conclusion}

In summary, infertile women with repeated IVF-ET have a low hope level. Maintaining a good marriage adjustment and establishing a good social support and relationship network could effectively improve their hope levels. It would be greatly appreciated if authorities were involved or some charitable funds were provide to financially support repeated IVF-ET treatments, particularly for those with low monthly incomes. As medical practitioners, we should take the initiative to undertake a variety of targeted nursing interventions to better enhance the hope level of infertile women with IVF-ET. Especially for those with repeated IVF-ET cycles or low HHI scores, we can better improve their hope levels and the success rates of assisted pregnancy by guiding them and their families, encouraging them to actively seeking more social support and receive more attention from their spouses.

\section{Abbreviations \\ IVF-ET: In vitro fertilization-embryo transfer; HHI: Herth hope index; COS: Con- trolled ovarian stimulation; MAT: Marital adjustment test; SSRS: Social Support Rating Scale.}

\section{Acknowledgements}

The authors would like to thank all the participants who voluntarily participated in this study and research assistants who performed the data collection.

\section{Authors' contributions}

NY made the contributions on study design, data collection, analysis, interpretation, and wrote the paper, and HLM contributed equally to this article as the co-author. FQ provided guidance in the study design, and is the corresponding author. TCY made contributions on investigation execution and data analysis. QW assisted with the data interpretation. All authors have read and approved the final manuscript.

\section{Funding}

Shanghai Jiao Tong University School of Medicine: Nursing Development Program.

\section{Availability of data and materials}

The datasets used and/or analysed during the current study are available from the corresponding author on reasonable request.

\section{Declarations}

Ethics approval and consent to participate

All patients provided their informed written consent. The study was approved by the Ethics Committee of Shanghai Ruijin Hospital and was conducted in accordance with the principles of the Declaration of Helsinki.

\section{Consent for publication}

Not applicable.

\section{Competing interests}

The authors declare that they have no competing interests.

\section{Author details}

${ }^{1}$ Department of Nursing, Ruijin Hospital, Shanghai Jiaotong University School of Medicine, Shanghai, China. ${ }^{2}$ Reproductive Medical Center, Ruijin Hospital, Shanghai Jiaotong University School of Medicine, Shanghai, China. ${ }^{3}$ School of Nursing, Shanghai Jiaotong University, Shanghai, China.

Received: 19 July 2021 Accepted: 25 September 2021

Published online: 09 October 2021

\section{References}

1. Schmidt L, Holstein B, Christensen U, et al. Does infertility cause marital benefit? An epidemiological study of 2250 women and men in fertility treatment. Patient Educ Couns. 2005;59(3):244-51.

2. Nelson CJ, Shindel AW, Naughton CK, Ohebshalom M, Mulhall JP. Prevalence and predictors of sexual problems, relationship stress, and depression in female partners of infertile couples. J Sex Med. 2008;5:1907-14.

3. Coeffin-Driol C, Giami A. The impact of infertility and its treatment on sexual life and marital relationships: review of the literature. Gynecol Obstetr Fertil. 2004;32:624-37.

4. Khademi A, Alleyassin A, Amini M, Ghaemi M. Evaluation of sexual dysfunction prevalence in infertile couples. J Sex Med. 2008:5:1402-10.

5. Drosdzol A, Skrzypulec V. Quality of life and sexual functioning of Polish infertile couples. Eur J Contracept Reprod Health Care. 2008;13:271-81.

6. Tan S, Hahn S, Benson S, Janssen OE, Dietz T, Kimmig R, Hesse-Hussain J, Mann K, Schedlowski M, Arck PC, Elsenbruch S. Psychological implications of infertility in women with polycystic ovary syndrome. Hum Reprod (Oxford, England). 2008;23:2064-71.

7. Wischmann T. Implications of psychosocial support in infertility - a critical appraisal. J Psychosom Obstet Gynaecol. 2008;29:83-90.

8. Cousineau TM, Domar AD. Psychological impact of infertility. Best Pract Res. 2007;21:293-308.

9. Noorbala AA, Ramazanzadeh F, Malekafzali H, Abedinia N, Forooshani AR, Shariat $M$, Jafarabadi M. Effects of a psychological intervention on depression in infertile couples. Int J Gynaecol Obstet. 2008;101:248-52.

10. Benyamini Y, Gozlan M, Kokia E. Women's and men's perceptions of infertility and their associations with psychological adjustment: a dyadic approach. Br J Health Psychol. 2009;14:1-6.

11. Chachamovich JR, Chachamovich $\mathrm{E}$, Ezer $\mathrm{H}$, et al. Investigating quality of life and health-related quality of life in infertility: a systematic review. J Psychosom Obstetr Gynaecol. 2010;31(2):101-10.

12. Simonstein F, Mashiach-Eizenberg M, Revel A, et al. Assisted reproduction policies in Israel: a retrospective analysis of in vitro fertilization-embryo transfer. Fertil Steril. 2014;102(5):1301-6. https://doi.org/10.1016/j.fertn stert.2014.07.740.

13. Awtani M, Kapoor GK, Kaur P, et al. Anxiety and stress at different stages of treatment in women undergoing in vitro fertilization-intracytoplasmic sperm injection. J Hum Reprod Sci. 2019;12(1):47-52. https://doi.org/10. 4103/jhrs.JHRS_23_18.

14. Wei SU, Xiao-dan LI, Wen YE. Correlation between adverse emotions and social supports in patients with threatened abortion after in vitro 
fertilization and embryo transfer. Nurs Pract Res. 2020;17(5):90-2. https:// doi.org/10.3969/j.issn.1672-9676.2020.05.035.

15. Fradelos E, Tzavella F, Koukia E, et al. Integrating chronic kidney disease patient's spirituality in their care: health benefits and research perspectives. Mater Sociomed. 2015;27(5):354-8. https://doi.org/10.5455/msm. 2015.27.354-358.

16. Melo GAA, Silva RA, Silva MFC, Galvão MTG, Silva VM, Caetano JÁ. Religiosity and hope in patients with chronic renal failure: coping strategies. Int Arch Med. 2016;9(133):1-9. https://doi.org/10.3823/2004.

17. Yadav S. Perceived social support, hope, and quality of life of persons living with HIV/AIDS: a case study from Nepal. Qual Life Res. 2010;19:157-66.

18. Davison SN, Simpson C. Hope and advance care planning in patients with end stage renal disease: qualitative interview study. BMJ. 2006;333(7574):886-9. https://doi.org/10.1136/bmj.38965.626250.55.

19. Sahaf R, Sadat Ilali E, Peyrovi H, Akbari Kamrani AA, Spahbodi F. Uncertainty, the overbearing lived experience of the elderly people undergoing haemodialysis: a qualitative study. Int I Community Based Nurs Midwifery. 2017;5(1):13-21.

20. Bravin AM, Trettene ADS, Andrade LGM, Popim RC. Benefits of spirituality and/or religiosity in patients with chronic kidney disease: an integrative review. Rev Bras Enferm. 2019;72(2):541-51. https://doi.org/10.1590/ 0034-7167-2018-0051.

21. Rahimipour M, Shahgholian N, Yazdani M. Effect of hope therapy on depression, anxiety, and stress among the patients undergoing hemodialysis. Iran J Nurs Midwifery Res. 2015;20(6):694-9. https://doi.org/10. 4103/1735-9066.170007.

22. Charan J, Biswas T. How to calculate sample size for different study designs in medical research? Indian J Psychol Med. 2013;35(2):121-6.

23. Herth K. Abbreviated instrument to measure hope: development and psychometric evaluation. J Adv Nurs. 1992;17(10):1251-9.

24. Locke H, Wallace K. General marital satisfaction and adjustment scales. In: Fredman N, Sherman R, editors. The handbook of measurements for marriage and family therapy. New York: Brunner-Mazel; 1997.

25. Shuiyuan $X$. Theoretical foundation and research application of social support rating scale. J Clin Psychol Med. 1994;02:98-100.

26. Nan T, Yanhui Z, et al. Level of hope of infertile women during treatment and its influence factors: a 221-case study. J Nurs. 2018;25(06):28-32. https://doi.org/10.16460/j.issn1008-9969.2018.06.028.

27. Ying N, Chenye T, Chichi H. Psychological experience of patients undergoing in vitro fertilization-embryo transfer: a qual-itative study. Nurs J Chin People's Liberation Army. 2017;34(3):26-9. https://doi.org/10.3969/j. issn.1008-9993.2017.03.006.

28. Nayeri ND, Goudarzian AH, Herth $\mathrm{K}$, et al. Construct validity of the herth hope index: a systematic review. Int J Health Sci (Qassim). 2020;14(5):50-7.

29. Durgun Ozan Y, Okumuş H. Effects of nursing care based on Watson's theory of human caring on anxiety, distress, and coping, when infertility treatment fails: a randomized controlled trial. J Caring Sci. 2017;6(2):95109. https://doi.org/10.15171/jcs.2017.010.

30. Bashiri A, Halper Kl, Orvieto R. Recurrent Implantation Failure-update overview on etiology, diagnosis, treatment and future directions. Reprod Biol Endocrinol. 2018;16(1):121. https://doi.org/10.1186/ s12958-018-0414-2.

31. Kim HO, Sung N, Song IO. Predictors of live birth and pregnancy success after in vitro fertilization in infertile women aged 40 and over. Clin Exp
Reprod Med. 2017;44(2):111-7. https://doi.org/10.5653/cerm.2017.44.2. 111.

32. Kong L, Shao Y, Xia J, et al. Quantitative and qualitative analyses of psychological experience and adjustment of in vitro fertilization-embryo transfer patients. Med Sci Monit. 2019;25:8069-77. https://doi.org/10. 12659/MSM.916627.

33. $\mathrm{NiY}$, Tong $\mathrm{C}$, et al. The analysis of fertility quality of life and the influencing factors of patients with repeated implantation failure. Health Qual Life Outcomes. 2021;19(1):32. https://doi.org/10.1186/s12955-021-01666-3.

34. Yang L. Influence of psychological intervention on negative emotions and pregnancy outcomes in patients with repeated IVF-ET cycles. Chin Rem Clin. 2019;19(19):3445-7. https://doi.org/10.11655/zgywylc2019.19. 092.

35. Juan S, Min W. Effect of shared decision-making intervention on level of hope and compliance to intervention in patients with in vitro fertilization-embryo transfer. J Clin Med Pract. 2020;24(4):73-6, 80.

36. Lau B, Yao SH, Tam M, et al. Gratitude in infertility: a cross-sectional examination of dispositional gratitude in coping with infertility-related stress in women undergoing IVF. Hum Reprod Open. 2019;2019(3): hoz012. https://doi.org/10.1093/hropen/hoz012.

37. Yanghua W. Study on feasibility of Chinese version of Herth hope index for cancer patients. Chin Nurs Res. 2010;1:20-1. https://doi.org/10.3969/j. issn.1009-6493.2010.01.008

38. Redlich-Amirav D, Ansell LJ, Harrison M, et al. Psychometric properties of Hope Scales: a systematic review. Int J Clin Pract. 2018;72(7): e13213. https://doi.org/10.1111/ijcp.13213.

39. Gozuyesil E, Karacay Yikar S, Nazik E. An analysis of the anxiety and hopelessness levels of women during IVF-ET treatment. Perspect Psychiatr Care. 2020;56(2):338-46. https://doi.org/10.1111/ppc.12436.

40. Zhou FJ, Cai YN, Dong YZ. Stress increases the risk of pregnancy failure in couples undergoing IVF. Stress. 2019;22(4):414-20. https://doi.org/10. 1080/10253890.2019.1584181.

41. Talaulikar VS, Arulkumaran S. Maternal, perinatal and long-term outcomes after assisted reproductive techniques (ART): implications for clinical practice. Eur J Obstet Gynecol Reprod Biol. 2013;170:13-9.

42. Hilbert SM, Gunderson S. Complications of assisted reproductive technology. Emerg Med Clin North Am. 2019;37(2):239-49. https://doi.org/10. 1016/j.emc.2019.01.005.

43. Richards A, Boogert T, Livingstone M, Dalrymple C. Endometrioid ovarian carcinoma in a woman with a history of infertility and multiple in vitro fertilization treatment cycles. Ultrasound Obstet Gynecol. 2012;40(1):112-4.

44. Athanasios $P$, Nausheen $M$. The risk of poor ovarian response during repeat IVF. Reprod Biomed Online. 2021;42(4):742-7. https://doi.org/10. 1016/j.rbmo.2020.12.001.

45. Elder K, Mathews T, Kutner E, Kim E, Espenberg D, Faddy M, Gosden R. Impact of gonadotrophin stimulation for assisted reproductive technology on ovarian ageing and menopause. Reprod Biomed Online. 2008;16:611-6.

\section{Publisher's Note}

Springer Nature remains neutral with regard to jurisdictional claims in published maps and institutional affiliations.

\footnotetext{
Ready to submit your research? Choose BMC and benefit from:

- fast, convenient online submission

- thorough peer review by experienced researchers in your field

- rapid publication on acceptance

- support for research data, including large and complex data types

- gold Open Access which fosters wider collaboration and increased citations

- maximum visibility for your research: over 100M website views per year
}

At $\mathrm{BMC}$, research is always in progress.

Learn more biomedcentral.com/submissions 